II.

\title{
Beitrag zum Kaiserschnitte und über das Verhalten des bei demselben verwendeten Nahtmateriales.
}

Vou

\author{
Dr. F. Münchmeyer, \\ I. Assistenzarzt.
}

(Mit 3 Abbildungen.)

Seit der letzten Arbeit über die Kaiserschnittsoperationen an der Königl. Frauenklinik in Dresden ${ }^{1}$ ) musste an weiteren 6 Frauen der Kaiserschnitt vorgenommen werden, 3 Mal mit, 3 Mal ohne Erhaltung der Gebärmutter. Zur Veröffentlichung dieser 6 Fälle, denen sich ein weiterer 7., nach Porro operirt und in der genannten Arbeit Leopold's nicht berïcksichtigt, anschliesst, veranlassten mich zwei Gründe: einmal wollte ich die in der Klinik vorgenommenen Porro'schen Operationen zusammenhängend besprechen und die Berechtigung dieser Operation für ganz bestimmte Fälle durch unsere Beobachtung stützen, zu zweit bin ich in der Lage, einen anatomischen Beitrag zu den Veränderungen der Gebärmutter nach Vornahme des e rhal te n d en Kaiserschnittes $z u$ geben und dabei das von uns bisher bevorzugte Nahtmaterial einer Prüfung zu unterziehen.

Ueber die 3 erhaltenden Kaiserschnitte fasse ich mich kurz, zumal da der eine derselben (Nr. 27) schon durch Leopold ${ }^{2}$ ) genauere Berücksichtigung fand. Ich lasse die Krankengeschichten in knapper Darstellung folgen.

1 (Nr. XXVI der Leopold'schen Reihenfolge): Erstgebärende, lumbosacralky photisches, besondersim Ausgange quer verengtes Becken. Bedingte Anzeige. Lebendes Kind. Genesung.

1) Leopold, dieses Archiv, Bd. XXXIV, Hft. 2.

2) Dieses Archiv, Bd. XXXVI, Hft. 3. 
Am 14. Januar 1889 wurde die 25 jährige Erstgebärende, Frau M. aus Pirna, in die Klinik als Hausschwangere aufgenommen. Sie ist $150 \mathrm{~cm}$ gross, von schwächlichem Körperbau und zeigt auffallende Veränderungen an der Wirbelsäule (ein Bruder soll gleichfalls ,ausgewachsen" sein). Ihr Leiden begann im zehnten oder zwölften Lebensjahre. Die Wirbelsänle ist in dem oberen Abschnitte regelmässig gebildet, in der Höhe des zehnten Brustwirbels besteht eine starke Lordose, vom elften und zwölften Brustwirbel an beginnt eine kyphotische Verbiegung, welche am stärksten in der Gegend zwischen dem dritten Lendenwirbel und ersten Kreuzbeinwirbel ansgesprochen ist. Ueber der hervorragendsten Stelle des Kreuzbeines liegt eine gänseeigrosse, weiche, undeutlich schwappende, bewegliche Geschwulst, welche als ein Lipom angesprochen wird. Die Gegend der Hinterbacken ist beiderseits abgeflacht, beim Gehen, besonders nach rückwärts, erfolgt ein deutliches Uebereinandersetzen der Füsse. Mässiger Hängebauch, gesunde innere Organe. Das Becken zeigt so bedeutende Veränderungen, dass ich dasselbe demnächst besonders zu beschreiben gedenke; mit Rücksicht daranf mache ich hier nur kurze Angaben. Spinae $25^{1} / 2$, Cristae $251 / 2$, Trochanteres 28, Conjugata externa 20. Vorberg nicht zu erreichen. Entfernung der Tubera ossis ischii 6, der Spinae posteriores superiores 8 , der Steissbeinspitze bis zur Schamfuge $9^{1 / 2}$, der Kreuzbeinspitze bis dahin $13 \frac{1}{2}$, der Steissbeinspitze bis zum Tuber ossis ischii 8. Schambogen auffallend eng, Steissbein wenig beweglich.

Wehenbeginn am 28. Januar. Der Kopf steht wenig beweglich im Beckeneingange, der Scheidentheil ist noch nicht ganz verstrichen, der Muttermund geschlossen, I. Schädellage b, lebendes Kind. Die W.ehen bleiben an den beiden nächsten Tagen noch sehr schwach, erst am 31. Januar werden sie stärker und erreichen im Laufe des Tages ihre volle Kraft. Dadurch wird zwar der Kopf in den Beckeneingang fest hineingepresst, doch kann er bei der bedeutenden Verengerung im unteren Beckenabschnitte nicht tiefer treten; als sehr: ungünstig kommen noch die Einstellung des Kopfes in II. Gesichtslage und der immer noch nicht ganz verstrichene Scheidentheil hinzu. An eine natürliche Entbindung ist nicht zu denken; auch bei völlig erweitertem Muttermunde wïrde ein so kleines Kind, wie das vorliegende (es wird auf etwa $2600 \mathrm{~g}$ geschätzt), unzerkleinert den Beckenausgang nie überwinden. Es bleibt demnach nur die Wahl zwischen der Enthirnung des vollkommen lebensfrischen Kindes, beziehentlich nachdem bis zum Absterben gewartet wurde -- und das hätte noch lange dauern können —, oder dem Kaiserschnitte. Bei dem lebhaften Wunsche der Mutter selbst nach einem lebenden Kinde wird der Kaiserschnitt beschlossen.

Operation am 31. Januar, Nachmittags 7 Uhr; Dauer 49 Minuten. Unter Anwendung des Schlauches, wird die Operation in der schon oft beschriebenen Weise vorgenommen, die Gebärmutter mit 7 tiefen Chromsäurecatgut- und 13 oberflächlichen Seidennähten gesehlossen. Das Kind, ein Mädchen von $2620 \mathrm{~g}$ Gewicht und $48 \mathrm{~cm}$ 
Länge, 9 beziehentlich $7 \frac{1}{2} \mathrm{~cm}$ Kopfbreite, $33^{1 / 2} \mathrm{~cm}$ horizontalem Kopfumfange, schreit gleich nach dem Hervorziehen aus der Gebärmutter. Eine beim Anschneiden der letzteren entstandene, 2 cm lange Schnittwunde dicht uiber dem linken Knie des Kindes wird durch 2 Nähte vereinigt. Wochenbett bezïglich der Operation vollkommen ohne Störung, doch in den ersten Tagen viele Beschwerden durch eine infolge Katheterisiren entstandene Blasenentzündung und einige Male Temperatur bis 38,5 . Am 22. Tage Entlassung. Bauchwunde fest verheilt, Gebärmutter richtig gelagert und gut beweglich, nirgends der Bauchwand anliegend. Kind anfänglich von einer Amme, später mit der Flasche ernährt, gedieh gut, hatte bei der Entlassung $440 \mathrm{~g}$ zugenommen.

2 (Nr. XXVII). Zweitgebärende. Allgemein verengt platt-rhachitisches Becken (vera $61 / 2$ bis $7 \mathrm{~cm}$ ). Bedingte Anzeige. Lebendes Kind. Tod derMutter am siebenten Wochenbettstage an Verblutung in die Bauchhöhle (vgl. Le opold, dieses Archiv, Bd. XXXVI, Hft. 3).

3 (Nr. XXVIII). Drittgebärende. Allgemein verengt platt-rhachitisches Becken. Bedingte Anzeige. Lebendes Kind. Genesung.

25 jährige B., aufgenommen in die Klinik als Hausschwangere am 3. September 1889, war schon zwei Mal in der Anstalt entbunden worden; das erste Mal durch Enthirnung des lebenden Kindes mit nachfolgender Kranioklasie, das zweite Mal durch Wendung auf die Füsse; das Kind wurde zwar lebend entwickelt, trug aber eine Verletzung des Brustkorbes davon. Letzte Periode Mitte December vorigen Jahres. Gesunde, kräftige Frau, $149 \mathrm{~cm}$ gross, mit allgemein verengtem platt-rhachitischem Becken, Spinae $221 \%$, Cristae 25 , 'Trochanteres 30 , Conjugata externa 18, diagonalis $91 / 2$, vera $71 / 2 \mathrm{~cm}$.

Wehenanfang angeblich am 7. October; Kopf auf die linke Darmbeinschaufel abgewichen. Die Wehen lassen wieder nach und treten am nächsten Tage (8. October) von neuem ein. I. Schädellage, Kopf ganz beweglich über dem Beckeneingange. Herztöne gut. Wehen sehr schwach. Unter nur langsam sich verstärkenden Wehen erreicht am 10. October, also am dritten Tage nach dem Wehenanfange, der Muttermund die Grösse von $7 \mathrm{~cm}$. Bald erreichen die Wehen ibre volle Stärke, doch gelingt es ihnen nicht, den Kopf auch nur etwas in das kleine Becken hineinzupressen. Bis zum Abende - der Blasensprung war noch njcht erfolgt - wird die Wirkung der zuletzt sehr kräftigen Wehen abgewartet, dann aber, da gar keine Fortschritte in der Geburt eintreten, der Kaiserschnitt beschlossen. Ein Versuch der Wendung auf die Füsse hätte gewiss auch seine Berechtigung gehabt, doch liessen die Verletzungen bei der vor zwei Jabren vorgenommenen gleichen Operation (damals wog das Kind 3100 g) wenig Erfolg erwarten; man hätte möglicherweise das grosse, völlig lebensfrische Kind geopfert, während man es durch den Kaiserschnitt mit grosser Gewissheit retten konnte. 
Kaisersehnitt am 10. Oetober 1889, 10 Uhr 38 Minuten Abends. Unter Anwendung des Schlauches wird die Operation in der gewöhnlichen Weise vorgenommen, die Gebärmutterwunde durch neun tiefe Chromsäurecatgut- und durch oberflächliche Seidenknopfnähte vereinigt. Die Operation wird durch mehrfache Störungen in der Narkose erschwert, auch zeigt die Gebärmutter nach Schluss der Bauchw unde - vorher war kein Tropfen Blut aus den Nahtstellen herausgekommen - starke Neigung, zu erschlaffen, so dass sogar eine stärkere Blutung aus der Scheide durch $40^{\circ}$ heisse Carbolausspülungen, mit Erfolg, bekämpft werden muss. Der Schlauch lag infolge der mannigfachen Störungen 21 Minuten lang. Das Kind, ein Mädchen von $3340 \mathrm{~g}$ Gewicht und $50 \mathrm{~cm}$ Länge (Kopfbreite $9 \frac{1}{2}$, bez. $9 \mathrm{~cm}$ ), schrie sofort; wurde anfänglich von einer Amme, vom vierten Tage an schon von der Mutter selbst gestillt und zeigte bei der Entlassung eine Gewichtszunahme von $760 \mathrm{~g}$. Wochenbett vollkommen fieberlos; Entlassung am 23. Tage nach der Operation mit vollkommen fester Narbe. Gebärmutter mit der vorderen Bauchwand verwachsen.

Somit sind bis jetzt in der Dresdener Klinik 28 erhaltende Kaiserschnitte ausgeführt worden; von den Müttern starben $3=10,7$ Proc. (bei der letzten Berechnung aus 25 Operationen betrug die Sterblichkeit 8 Proc.). Von den 28 Kindern starb während des Aufenthaltes in der Klinik nur 1, während $27=$ 96,4 Proc. lebend entlassen wurden.

Diesen 28 Kaiserschnitten mit Erhaltung der Gebärmutter stehen 7 Operationen mit Abtragung derselben gegenüber. Die 3 ersten wurden seiner Zeit in der Schrift über den Kaiserschnitt ${ }^{1}$ ) durch Leopold veröffentlicht, 4 neue füge ich hinzu. Der besseren Uebersicht wegen schicke ich den Krankengeschichten der letzteren zunächst noch einmal kurze Angaben über die 3 ersten Fälle voraus.

1. Frau P., 37 Jahre alt, viertgebärend. Hochgradig all gemein verengtes platt-rhachitisches Becken, mit einer Vera von etwa $5 \mathrm{~cm}$. Tetanie der Gebärmutter, starke Quetschung und Entzündung des Gebärmutterhalses, Misslingen der Wendung, Blasensprung $2 / 2$ Tage vor der Operation. Operation am 4. Januar 1885, Abtragung der Gebärmutter und der Anhänge, Einnähung des mit dem $\mathrm{P}$ a quelin' schen Brenner verschorften Stumpfes in den unteren Wundwinkel. Kind tief scheintodt, stirbt 16 Stunden nach der Geburt an Leberzerreissung. Die Frau wurde am 68. Tage mit kleiner granulirender Wundfäche und bei sehr gutem Allgemeinbefinden entlassen.

1) Der Kaiserschnitt and seine Stellang u. s. w., S. 146 u. 147. Stuttgart 1888. 
2. Frau B., 33 Jahre alt, fünftgebärend. Primäres, dickknolliges Carcinom der Scheide mit Infiltration des linken Beckenabschnittes, Verlegung der weichen Geburtswege. Scheidentheil vollkommen gesund. Operation am 8. December 1885 ohne Besonderheiten.' Lebendes Kind. Mutter und Kind mit gutem Allgemeinbefinden am 47. Tage entlassen.

3. Frau St., 32 Jahre alt, fünftgebärend. Weit vorgeschrittenes Carcinom des Gebärmutterhalses, zerklüftet und mit verhärteten Rändern auf der linken Seite. Völlige Undurchgängigkeit des Mutterhalses, enges Becken (vera $8 \mathrm{~cm}$ ). Operation am 16. Februar 1886 ohne Besonderheiten. Lebendes Mädchen; starb am 30. Tage an eitriger Nabelentzündung. Bei der Mutter trat am 14. Wochenbettstage Geistesstölung infolge chronischen Alkoholismus ein, welche am 33. Tage nach der Operation die Verlegung auf die Irrenabtheilung des städtischen Krankenhauses erforderte.

\section{Neue Fälle:}

4. Erstgebärende. Hochgradig allgemein verengt platt-rhachitisches Becken. Lange Geburtsdauer, abgestorbenes Kind; starker Eiweissgehalt des Harnes. Genesung.

Am 11. December 1888 wurde die 28 jährige $H$. in die Klinik aufgenommen. Sie hat im zweiten Isebensjahre laufen gelernt, giebt an, an englischer Krankheit gelitten zu haben und brach infolge derselben im sechsten Jahre den rechten, im achten den linken Oberschenkel. Letzte Periode am 15. März. Kleine, $136 \mathrm{~cm}$ messende, schwächliche Person. Geringe Skoliose der Lendenwirbelsäule. Beide Oberschenkel sind nach vorn und rechts verbogen, das rechte Bein bedeutend kürzer, als das linke; das rechte Knie steht bei vollkommen gerader Rückenlage um $4 \mathrm{~cm}$ höher als das linke. Die linke Hüfte steht höher als die rechte; die linke Spina superior anterior mindestens $2 \mathrm{~cm}$ über der rechten. Beide Darmbeinschaufeln sind bedeutend verkleinert, steil, nach aussen umgekippt. Die Darmbeinkämme fallen kurz nach hinten ab. Beckenmaasse: Spinae 24\%, Cristae 23, Trochanteres $31 \%$, Conjugata externa 15 , diagonalis $71 / 2$ bis 8 , vera $5 \%$ bis $6 \mathrm{~cm}$. Vorberg springt stark vor; von der Mitte der Schamfuge bis zum rechten oberen Hüftbeinstachel $14 \%$, bis zum linken $16^{1} / 2$, von der Kreuzbeinspitze bis zum unteren Rande der Schamfuge 9, von der Steissbeinspitze bis dahin $6^{1 / 2}$, von einem Sitzknorren bis zum anderen $10^{1 / 2} \mathrm{~cm}$. Im Harne etwa $1 / 3$ Volumen Eiweiss. Die Wehen sollen zwei Tage vor dem Eintritte in die Klinik begonnen haben. Die Gebärmutter hat die Form einer umgekehrten 8; in der Kappe des Mutterhalses, welche sich durch eine ziemlich deutliche Furche von dem oberen Gebärmutterabschnitte abhebt, liegt der grosse kindliche Kopf, welcher sich mit dem rechten Scheitelbeine (I. Schädellage a) über der Schamfuge stark vorwölbt. Kind lebt. Durch den für die Fingerspitze durchgängigen Muttermund stülpt sich die Blase wurstförmig in die Scheide vor. J Leichte 
Wehen, sind am nächsten Tage noch nicht viel kräftiger; am Abende kindliche Herztöne noch deutlich gehört. Plötzlich wird in der Nacht der dienstthuende Arzt von der Hebamme benachrichtigt, dass sie die Herztöne nicht mehr höre; auch er findet dieselben vollkommen erloschen. Zwei Entbindungsarten konnten in Frage kommen: die Enthirnung und der Kaiserschnitt; von der ersteren waren kaum günstige Erfolge zu erwarten, der Muttermund war nur markstückgross, bis zu der nothwendigen Erweiterung hätte noch lange Zeit vergehen müssen; es erheischte aber die lange Geburtsdauer, der 'Tod der Frucht, der starke Eiweissgehalt des Harnes eine möglichst baldige Entbindung. Diese konnte nur der Kaiserschnitt ermöglichen. Das Kind war abgestorben, die Gefahr der Zersetzung der Eitheile und dadurch anch der Gebärmutterschleimhaut waren nicht von der Hand zu weisen; so musste auf die Erhaltung der Gebärmutter verzichtet werden.

Operation am 12. December 1888, Nachmittags 4 Uhr. Gründliche äusserliche Reinigung und Desinfection der Frau, Auswischen der Scheide mit Sublimatlösung (1:4000), Ausstopfung mit Jodoformgaze. In tiefer Narkose wird die Bauchböhle durch einen vom Nabel bis zur Schamfuge reichenden Schnitt eröffnet, die Gebärmutter vorgezogen und unterhalb des Kopfes und der Gebärmutteranhänge ein mittelstarker Gummischlauch zwei Mal fest umgelegt; zwei sich kreuzende Seidennähte sichern den Knoten. Tiefe Nähte vereinigen oberhalb und unterhalb der Gebärmutter die Bauchdecken - dadurch wird am sichersten eine Verunreinigung der Bauchhöhle durch das bei der Abtragung ausfliessende Blut, Fruchtwasser u. ä. vermieden - , die Gebärmntter wird durch allseitig fest angedrückte Schwämme umgeben und dann oberhalb des Schlauches in einem Zuge quer abgetragen. Kind und Fruchtkuchen werden entfernt, die Serosa der Bauchdecken rings um den Stumpf vorgezogen, peinlichst mit in Sublimatlösung (1:5000) getauchten Schwämmen abgewischt und durch Seidennähte unterhalb des Schlauches mit dem Bauchfelle der Gebärmutter vernäht; an der rechten Seite gelingt das nicht und muss hier die Vernähung über dem Schlauche erfolgen. Eine quer durchgestossene Spicknadel sichert den Stumpf vor dem Herabgleiten; letzterer wird möglichst gekürzt, die Gebärmutterschleimhaut trichterförmig ausgeschnitten und die so gesetzte Wunde sowohl, als auch der ganze Stumpf mittels des Paquelin'schen Brenners verschorft. Oberflächliche Bauchdeckennähte, Druckverband mit Jodoform- und Sublimatgaze und Watte. Dauer der Operation 1 Stunde 15 Minuten, Das Kind, ein todtes Mädchen, war $49 \mathrm{~cm}$ lang, $2400 \mathrm{~g}$ schwer, mit Kindespech über und iiber bedeckt; linkes Scheitelbein unter das rechte geschoben (Querdurchmesser $81 / 4$, bez. $7 \mathrm{~cm}$ ), Eihäute und Gebärmutterschleimhaut grïnlich-grau verfärbt. Wochenbett vollkommen regelmässig bis auf eine einmalige Temperatursteigerung von $38,5^{\circ}$ bei 106 Pulsen. Am achten Tage die oberflächlichen, am zwölften die tiefen Bauchdeckennähte nebst Spicknadel entfernt; am 1. Januar der Schlauch gelöst und die Bauchfellnähte am Stumpfe berausgenommen, nachdem bei jedem Verbandwechsel 
der durch Aufstreuen eines Pulvers von Tannin und Salol zu gleichen Theilen trocken gehaltene Stumpf möglichst gekürzt worden war. Am 24. Januar, dem 43. 'Tage nach der Operation, in bester Gesundheit und mit fester Bauchnarbe entlassen.

5. Erstgebärende. Allgemein verengt platt-rhachitisches Becken. Lange Geburtsdauer; lebendes Kind. Genesung.

17jährige M., aufgenommen als Haussehwangere in die Klinik am 19. April 1889; hat an englischer Krankheit gelitten, weiss nicht, wann sie laufen lernte, sonst immer gesund. Letzte Periode im Juli 1888. $145 \mathrm{~cm}$ gross, mässig kräftig, mit gesunden inneren Organen. Allgemein verengtes, platt-rhachitisches Becken: Spinae $22 \%$, Cristae 25 , Trochanteres $29 \%$, Conjugata externa 17 , diagonalis $71 / 2$, vera $51 / 2 \mathrm{~cm}$; beide ungenannten Linien leicht abzutasten, Schambogen sehr eng, Vorberg stark vorspringend. Wehenanfang am 14. Mai; Muttermund für die Fingerspitze durchgängig, Kopf ganz beweglich über dem Beckeneingange. Auch am nächsten Tage noch schwache Wehen, welche den Muttermund sehr langsam erweitern. Am 16. Mai letzterer fünfmarkstïckgross, Blase prall gespannt. Ein Versuch, den bis jetzt noch immer vollkommen beweglich und hoch stehenden Kopf dadurch tiefer zu bringen, dass bei $7 \mathrm{~cm}$ weit eröffnetem Muttermunde die Blase gesprengt wird, hat keinen Erfolg. Unter Abfluss von reichlichem Kindespech tritt zwar anscheinend der Kopf tiefer, in Wirklichkeit wölbt sich aber nur die mehr und mehr wachsende Kopfgeschwulst nach der Scheide hin vor. Dabei werden die Herztöne immer undeutlicher and schliesslich ïberhaupt nicht mehr gehört. Von der Enthirnung wird bei der noch nicht völligen Erweiterung des Muttermundes und der beträchtlichen Enge der Scheide abgesehen, an eine natiurliche Geburt, beziehentlich an die Wendung ist - bei der hochgradigen Beckenenge nicht zu denken; es bleibt demnach als die einzige Entbindungsart nur der Kaiserschnitt übrig. Die lange, sich iber mehrere Tage erstreckende Geburtsdauer, die vielfachen Untersuchungen, der muthmasslich eingetretene Tod des Kindes lassen die Erhaltung der Gebärmutter gefährlich erscheinen; deshalb wird die Operation nach Porro beschlossen und am 16. Mai, 31/4 Uhr Nachmittags vorgenommen. Nach Eröffnung der Bauchhöhle wird die Gebärmutter vorgewälat, die Hand des Gehülfen umgreift fest den unteren Abschnitt derselben und die Gebärmutter wird durch einen Längenschnitt eröffnet. Der vorn sitzende Fruchtkuchen wird zur Seite geschoben, das Kind sofort hervorgezogen und abgenabelt; von Kindespech dicht bedeckt, ist es erst nach langen Wiederbelebungsversuchen zam Schreien zu bringen. Nach Entfernung der Nachgeburt wird der Schlauch umgelegt, und dann die Operation in der oben beschriebenen Weise beendet. Dauer 1 Stunde 20 Minuten. Das Kind, ein Mädchen, war $48 \mathrm{~cm}$ lang, wog $2800 \mathrm{~g}$; die Kopfbreite betrug $91 / 2$, beziehentlich $71 / 2 \mathrm{~cm}$.

Die ersten vier Wochenbettstage regelmässig, am fünften e k lam . ptischer Anfall, der sich im Laufe des 'Tages noch vier Mal 
wiederholte; von da ab war der Verlauf des Wochenbettes durchaus gut und fieberlos. Am 36. Tage nach der Operation vollkommen gesund entlassen. Das Kind war von Anfang an sehr elend. Ernährung anfänglich durch eine Amme, dann mittels der Flasche; andauernde, aller Behandlung trotzende Durchfälle führten am siebenten 'Tage den Tod des Kindes herbei. Die Leichenöffnung ergab: Allgemeine Blutleere, follikuläre Darmentzündung.

6. Erstgebärende. Allgemein verengtes rhachitisches Becken. Vollkommene Erschlaffung der Gebärmutter bei dem Versuche des erhaltenden Kaiserschnittes. Lebendes Kind. Genesung.

29 jährige S., aufgenommen als Hausschwangere am 12. Juni 1889. Die kleine $(135 \mathrm{~cm})$, sehr blutarme und schwächliche Person lernte erst im dritten Lebensjahre laufen. Letzte Periode am 25., Empfängniss am 27. December. Gesunde innere Organe; geringe Skoliose, die rechte Schulter steht etwas tiefer als die linke. Beckenmaasse: Spinae $221 / 2$, Cristae 24, Trochanteres $27^{1 / 2}$, Conjugata externa 14 , diagonalis $7^{3} / 4$, vera $5^{3 / 4} \mathrm{~cm}$. Vorberg stark vorspringend, ungenannte Linie beiderseits leicht abzutasten. Wehenanfang am 22. September. Kopf steht in II. Schädellage ganz beweglich ïber dem Beckeneingange; Muttermund markstückgross. Die anfänglich sehr schwachen Wehen werden bis zum Nachmittage stärker, können aber den Kopf nicht tiefer bringen. Bei der hochgradigen Beckenenge wird zur Rettung des vollkommen lebensfrischen Kindes der Kaiserschnitt beschlossen.

Die Operation (22. September, Abends 10 Uhr 13 Minuten) wird mit der Absicht, die Gebärmutter zu erhalten, begonnen: Im Anfange drückt die Hand des Gehülfen die vorgewälzte Gebärmutter in ihrem unteren Abschnitte zusammen, um 10 Uhr 16 Minuten wird nach Entfernung des sofort schreienden Kindes ein Schlauch umgelegt. Schon beim Anschneiden fiel die hochgradige Verdünnung des Gebärmuttermuskels auf. Die Dicke der Wand war so gering, wie wir sie bei keinem Kaiserschnitte früher gesehen hatten, und betrug auch nach der Entleerung und der darauf folgenden Zusammenziehung nur. wenige Millimeter. Die acht tiefen Chromsäurecatgut- und die oberflächlichen Seidennähte erfordern 16 Minuten, dann wird der Sehlauch gelockert. Sofort dringt aus allen Nahtstichen Blut hervor, " die Gebärmutter zieht sich trotz aller dahin zielenden Maassnahmen - Ergotin, Kneten, Aufdrücken heisser Schwämme - nicht zusammen. Bei der schwächlichen; blutarmen Person sind weitere Versuche nicht am Platze; kulz entschlossen, wird alsbald (10 Uhr 32 Minuten) der Schlauch von neuem festgezogen, der Knoten durch eine Seidennaht gesichert und die Gebärmutter abgetragen. Weiterer Verlauf der 1 Stunde 7 Minuten dauernden Operation ohne Besonderheiten. Das Kind, ein Mädchen, wog $2536 \mathrm{~g}$ und war $48 \mathrm{~cm}$ lang; Kopfbreite $8^{3} / 4$, bez. $7 \frac{1}{2} \mathrm{~cm}$. Es gedieh an der Ammenbrust sehr gut und wurde nach 14 Tagen mit $200 \mathrm{~g}$ Gewichtszunahme abgeholt. Wundverlauf rollkommen regel- 
mässig und fieberlos. Entlassung bei bestem Allgemeinbefinden am 43. Tage nach der Operation.

7. Eistgebärende. Weit vorgeschrittenes Carcinom des Gebärmutterhalses. Starer Muttermund. Todtes $\mathrm{K}$ ind.

Frau B., 38 Jahre alt, wurde am 9. October 1889 aufgenommen. Die 10 voraufgehenden Geburten waren natürliche, doch traten schon während đer beiden letzten Wochenbetten unregelmässige Blutungen auf. Letzte Periode am 7. Januar. Frau mässig kräftig, Becken wohlgebant, die todte Frucht liegt beweglich über dem Beckeneingange in II. Schädellage. 14 Tage nach der letzten Periode soll eine stärkere. Blutung eingetreten sein, welche sich in unregelmässigen Zwischenräumen wiederholte. Im letzten Vierteljahre nalımen die Blutungen $\mathrm{zu}$ und hörten in den beiden letzten Wochen vor der Aufnahme nicht mehr auf; dabei heftige Schmerzen. Jetzt geht bei jeder Wehe Blut ab. Beide Muttermundslippen sind stark zerklüftet, auffallend hart, bei Berihrung leicht blutend. Beide Parametrien starr infiltrirt, Leistendrüsen geschwollen, Gebärmutterhals sehr eng; beiderseits geht das Carcinom auf das Scheidengewölbe über.

Trotz kräftiger Wehen erweitert sich der starre, krebsig entartete Muttermund nicht; eine natürliche Erweiterung ist demnach kaum zu erwarten, auch liegt die Befürchtung nahe, dass eine Ausdehnung der starren Lippen, genïgend, eine reife Frucht hindurchzulassen, nicht ohne grosse Zerreissungen und starke Blutungen vor sich gehen würde; eine künstliche Erweiterung, stumpf oder blutig, konnte der schon durch die vorausgegangenen Blutungen geschwächten Frau das Leben kosten. So erschien der Kaiserschnitt als die immerhin schonendste aller Entbindungsarten; nur die Abtragung der Gebärmutter konrıte dabei in Frage kommen. Eine in ihren, unteren Theilen krebsig entartete Gebärmutter zu erhalten, ist zwecklos, die Abtragung aber und äussere Stielversorgung ist gegen die möglicherweise im Wochenbette eintretende Verjauchung der Krebsmassen der sicherste Schutz. Operation am 10. October in der gewohnten Weise. Dauer 36 Minuten. Die todte, zum Theil schon verweste Frucht wog $3200 \mathrm{~g}$ bei $52 \mathrm{~cm}$ Länge. Wundverlauf regelmässig. Der aashafte Geruch des Wochenflusses, von den zerfallenden Krebsmassen herrithrend, machte mehrfache desinficirende Ausspülungen nothwendig. Die Frau wird demnächst zur Entlassung kommen.

In 5 Jahren ist somit in der Dresdener Klinik 7 Mal der Kaiserschnitt mit Abtragung der Gebärmutter vorgenommen worden. Diesen 7 Fällen stehen 28 (also 1 zu 4) gegenüber, in denen die Gebärmutter erhalten werden konnte. Von den der Porro'schen Operation unterworfenen Frauen starb keine, alle verliessen mit geheilter Bauchwunde, bis auf eine, mit vollkommen zufriedenstellendem Allgemeinbefinden die Klinik. Diese eine ( $\mathrm{Nr}$. III) musste wegen Geistesstörung in das städtische 
Krankenhaus verlegt werden; sicherlich ist diese, am 14. Tage nach der Operation eintretende Störung nur ein Rückfall der dem chronischen Alkoholismus ergebenen Frau in ihr altes Leiden. Die geistige Störung steigerte sich nach der Verlegung immer mehr, schliesslich trat vollständige Nahrungsverweigerung ein. Dazu kam am Kreuzbeine und in der Gegend der Trochanteren Druckbrand und nach sechswöchentlichem Aufenthalte im Krankenhause erfolgte der Tod an Erschöpfung. Von Frau B. (Nr. II) war keine Nachricht zu erhalten, Frau P. (Nr. I) schrieb vor kurzem, dass sie sich sehr wohl befinde, Frau H. (Nr. IV) und M. (Nr. V) stellten sich kürzlich zur Untersuchung vor. Beide erfreuen sich eines vollkommen guten Allgemeinbefindens, die Bauchnarbe ist fest, keine Spur eines Bauchbruches vorhanden, vornehmlich fehlen alle Klagen, welche auf die Entfernung der Gebärmutter und ihrer Anhänge bezogen werden könnten.

Von den 7 Kindern waren 2 vor der Entbindung abgestorben, 1 kam scheintodt zur Welt und starb nach wenigen Stunden, 2 andere starben wenige Tage nach der Geburt, das eine an Nabel-, das andere an Darmentzïndung. Demnach konnten nur $2 \mathrm{Kin}-$ der die Klinik lebend verlassen.

In dem Müller'schen Handbuche der Geburtshülfe (Bd. III, S. 209) giebt Fehling mit Benutzung der Godson'schen Statistik an, dass in 10 nach P orro operirten Fällen mit intraperitonealer Stielversorgung 10, in 125 mit extraperitonealer Behandlung 67 Frauen starben, demnach von 135 Operirten $77=57$ Proc. Von den 7 in der Dresdener Klinik nur mit extraperitonealer Methode behandelten Frauen starb keine. Dieser 0 Proc. Sterblichkeit bei der Operation nach Por ro stehen 10,7 Proc. bei der erhaltenden Methode gegenüber. Es würde ein grosser Fehler sein, wenn man aus dieser Thatsache den Schluss ziehen wollte, die Porro'sche Operation verdiene vor der erhaltenden Methode den Vorzug. Von den 3 bei der letzteren eingetretenen Todesfällen ist der eine durch eine sicherlich schon vor der Operation entstandene Sepsis bedingt worden - hier hätte von vornherein die Abtragung der Gebärmutter erfolgen müssen -, in dem zweiten Falle war die Todesursache ein hochgradiger Meteorismus, verbunden mit Lungenödem, Zustände, welche bei der buckeligen Person wahrscheinlich in gleicher Weise auch nach der Abtragung der Gebärmutter eingetreten wären. Die dritte Frau erlag einer Blutung in die Bauchhöhle. Diese hätte die 
Abtragung der Gebärmutter verhindern können. So schrumpft die Sterblichkeitsziffer der erhaltenden Operation sehr zusammen, hier ein der Methode selbst zur Last fallender Todesfall, bei dem Porro'schen Verfahren keiner. Das ist von untergeordneter Bedeutung, schon der nächste nothwendig werdende Fall mit $\mathrm{Ab}$ tragung der Gebärmutter kann tödtlich verlaufen, und dann sind die Erfolge die gleichen. Sehen wir deshalb von dem Ausgange der Operation ganz ab, betrachten vielmehr nur die Gesammtfolgen für die Frauen, so sind dieselben bei der Porro'schen Methode viel ungünstiger. Bei dem erhaltenden Kaiserschnitte bleibt der Frau die Möglichkeit einer weiteren Empfängniss in einzelnen wenigen Fällen ist ja auch eine erneute Schwangerschaft eingetreten -, die Heilungsdauer ist eine kürzere, durchschnittlich 3 bis $3^{1 / 2}$ Wochen gegenüber den 6 Wochen, welche die mit Abtragung der Gebärmutter Operirten durchschnittlich in der Klinik zubringen mussten. Die anderen Nachtheile, welche man dem Porro'schen Verfahren nachsagt, die befürchteten Folgezustände nach Entnahme der Gebärmutter und Anhänge; weist bisher keine unserer Operirten auf, ebenso scheinen uns Bauchbrüche infolge der Einnähung des Stumpfes in die Bauchwände, besonders unter Berücksichtigung unserer Erfahrungen bei der Myomotomie mit extraperitonealer Stielversorgung, nicht häufiger zu sein, als nach anderen Bauchschnitten.

Wenn irgend möglich, soll man bei der. Vornahme des Kaiserschnittes die Gebärmutter zu erhalten suchen, nur dort, wo die Erhaltung der Gebärmutter für die Frau Gefahren mit sich bringt, ist die Entfernung geboten. Die Bedingungen zur Vornahme der Porro'schen Operation hat $\mathrm{Z}$ weifel in der neuesten Auflage seines Lehrbuches der Geburtshülfe folgendermaassen zusammengefasst: „Die Porro'sche Operation ist da am Platze, wo die Normaloperation mit Naht nicht mehr zu wagen ist. Solche Verhältnisse sind gegeben, wenn man ein septisch inficirtes Organ zurücklassen müsste, wo wegen Scheidennarben oder anderer Verhältnisse kein Abfluss der Lochien möglich wäre, wo grosse Geschwülste, Fibroide u. dgl. an der Gebärmutter sitzen, welche die Frau krank erhalten, ihr vielleicht eine neue Operation nothwendig machen, die vereitern können u. s. w." Fügen wir noch als weitere Anzeigen die Osteomalakie und die vollkommene Erschlaffung der Gebärmutter bei dem Versuche des erhaltenden 
Kaiserschnittes hinzu, so sind die Grenzen für das Porro'sche Verfahren gezogen, und diese soll man nicht überschreiten. Sehen wir, wie weit wir dieselben einhielten.

In dem ersten Falle (Nr.I) war sicherlich eine Infection der Frau eingetreten, Quetschung und Entzündung des Gebärmutterhalses nach lange dauernder Geburt bei engem Becken und misslungener Wendung hatten sie herbeigeführt. In den Fällen IV und $\mathrm{V}$ war die Infection zwar nicht mit Gewissheit eingetreten, die Wahrscheinlichkeit einer solchen aber nicht von der Hand zu weisen; somit wäre es ein leichtfertiges Spielen mit einem Menschenleben gewesen, hätte man die Gebärmutter bei der Wahrscheinlichkeit einer Entzündung versenken wollen. In solchen Fällen nehme man lieber der Frau die Möglichkeit einer weiteren Empfängniss - gross ist dieselbe ja auch bei dem erhaltenden Kaiserschnitte nicht - , als dass man sich zu einem gewagten Versuche herbeilasse. Bei so elenden körperlichen Verhältnissen wie in den beiden vorliegenden Fällen, hat man, wie es Braun in seinem Lehrbuche betont, auch gewisslich Recht, die Frauen durch die Entfernung der Gebärmutter von der Gefahr einer späteren Schwàngerschaft zu befreien.

In 3 weiteren Fällen (Nr. II, III und VII) gaben Carcinom den Anlass zur Vornahme des Kaiserschnittes nach Porro. Ist das Carcinom noch nicht so weit vorgeschritten, dass sich der Muttermund einigermaassen erweitern kann, so wird man bei abgestorbenem Kinde natürlich die Enthirnung vornehmen; das lebende Kind aber zu tödten, um ein verlorenes mütterliches Leben etwas weniger zu gefährden, das ist meiner Meinung nach ein Fehler. Erweitert sich der Muttermund nicht,- verlegen krebsige Infiltrate der Parametrien den Beckeneingang, wie in Fall II, dann ist es allein richtig, den Kaiserschnitt zu machen. Stumpf oder mit dem Messer sich einen Weg durch die Krebsmassen zu bahnen, erscheint wegen der unberechenbaren Blutungen gefährlicher, als die Vornahme des Kaiserschnittes, den die 3 von uns operirten Frauen gut überstanden. Macht man wegen Krebs den Kaiserschnitt, so soll, wenn nur irgend möglich, die Gebärmutter abgetragen werden; so verhindert man am sichersten die Gefahr einer Verhaltung des Wochenflusses und die möglicherweise noch nachträglich erfolgende Infection der Gebärmutter von den im Wochenbette zerfallenden Krebsmassen aus; jedes Mal wird die Abtragung nicht möglich sein, es können Fälle vorkommen (vergl. 
Teuffel, dieses Archiv, Bd. XXXVI, Hft 2), in denen der Krebs so weit vorgeschritten ist, dass sich der Schlauch nicht anlegen und ein Stumpf nicht bilden lässt; in solchen Fällen bleibt dann nur der erhaltende Kaiserschnitt mit sorgfältigst angelegter Naht übrig.

In dem letzten unserer Fälle schliesslich (Nr. VI) zwang eine hochgradige, nicht zu bekämpfende Erschlaffung der schon regelrecht vernähten Gebärmutter, den ursprünglichen Operationsplan zu ändern und doch noch die Gebärmutter abzutragen. Es sind in der Literatur in der jüngsten Zeit mehrfache Beobachtungen gleicher Art gemacht worden, und soll nach Schauta eine derartige Erschlaffung des Gebärmuttermuskels nicht selten die Folge einer zu lange dauernden und zu starken Umschnürung durch den Gummischlauch sein; eine Lähmung des Muskels könne durch eine derartige Umschnürung herbeigefuihrt werden, und sei es deshalb besser, den Schlauch möglichst zu vermeiden. Leopold sprach sich in seiner vorletzten Veröffentlichung über den Kaiserschnitt (dieses Archiv, Bd. XXXIV, Hft. 2) dagegen aus. Er glaubt, dass in den erwähnten Fällen die Umschnürung eine zu feste und die hervorgerufene Blutleere eine unnöthig grosse gewesen sei; lege man den Schlauch nicht zu fest um und löse ihn nach Anlegung der tiefen Nähte, so wäre eine bedrohliche Blutung nicht zu befürchten und in seinen Fällen auch noch nicht vorgekommen. Die neuerdings von uns gemachten Erfahrungen scheinen indessen diese Annahme docb nicht so ganz zu bestätigen. In dem Falle XXVII hatte der Schlauch 13, in Nr. XXVIII durch äussere Umstände 21 Minuten und schliesslich in Nr. VI der Porro-Operationen 16 Minuten. gelegen: der Schlauch war sicherlich nicht zu fest angezogen worden. In dem zweiten Falle (Nr. XXVIII) liess sich die Erschlaffung der Gebärmutter durch entsprechende Maassnahmen bekämpfen, in dem ersten trat eine tödtliche Nachblutung ein; wieviel Schuld auf die Naht, wieviel auf die Anwendung des Schlauches zu schieben ist, vermag ich nicht zu entscheiden. Bei Frau S. (Nr. V) konnte die Verblutungsgefahr noch durch die rechtzeitige Abtragung der schlaffen Gebärmutter beseitigt werden. Es mag ja sein, dass bei dieser schwächlichen und blutarmen Person bei der auffallenden Dünne der Gebärmutterwand die Wehenschwäche auch ohne die Anwendung des Schlauches eingetreten wäre, immerhin sind die drei Beobachtungen, zumal im 
Vereine mit denen anderer Operateure, eine ernste Mahnung, den Schlauch nicht zu lange und nicht zu fest anzulegen, ihn vielmehr lieber dort, wo geübter Beistand zur Verfügung steht, ganz zu vermeiden; wer nur mit einem Gehülfen operirt, wird allerdings die durch die Umschnürung in Anspruch genommene Hand nur ungern missen.

Bei der Besprechung des vierten unserer Kaiserschnitte nach Porro habe ich unser Operationsverfahren genauer beschrieben; wo es möglich ist, wird das Bauchfell unter dem Schlauche vereinigt; ist das zu schwierig oder unmöglich, so erfolgt die Vereinigung mit gleichem Erfolge auch über demselben. In den beiden letzten Fällen erleichterten wir uns die Vernähun unter dem Schlauche dadurch, dass wir zunächst das Bauchfell der Bauchdecken durch Nähte mit dem der Gebärmutter vereinigten und dann erst über der Naht den Schlauch anlegten. Der Stumpf wird durch eine lange, quer durchgeführte Spicknadel vor dem Hinabgleiten in die Tiefe gesichert; die Nadel wird meist mit den letzten tiefen Bauchnähten am 12. bis 14. Tage, der Schlauch nach etwa drei Wochen entfernt. So ist man vor einem Herabgleiten des Stumpfes in die Tiefe mit nachträglicher Infection der Bauchhöhle ziemlich sicher. Um den Stumpf trocken zu erhalten, wird bei jedem Verbandwechsel ein Gemisch von Tannin und Salol zu gleichen Theilen aufgestreut, jedesmal aber vorher eine möglichst ausgiebige Kürzung der abgestorbenen Massen mittels Scheere und Pincette vorgenommen. Die Frauen bleiben wenigstens vier Wochen im Bette und dürfen dasselbe nur mit einer gut angepassten und fest schliessenden Leibbinde verlassen. Die kürzeste Heilungsdauer betrug in unseren Fällen (Nr. III und VII sind dabei nicht berücksichtigt) 36 , die längste 68 Tage. Das ist ein gewiss recht grosser Nachtheil der extraperitonealen Stielversorgung, doch bietet diese solche Sicherheit der mehr als gefährlichen Versenkung des Stumpfes gegenüber, dass wir uns zu der letzteren schwerlich entschliessen werden. Von den anderen, dem Verfahren nachgesagten Folgen, wie leichterem Entstehen eines Bauchbruches, Zerrungserscheinungen durch den Stumpf, haben wir in unseren 7 Fällen nichts gesehen.

Um die mehr als gefährliche Versenkung des Stieles zu umgehen (nach der oben erwähnten Zusammenstellung Fehling's kamen auf 10 derartige Operationen 10 Todesfälle), andererseits um die Folgen der extraperitonealen Versorgung möglichst zu 
326 Münchmeyer, Beitrag zum Kaiserschnitte $u$, über das Verhalten

vermeiden, hat Frank (vergl. Beaucamp, dieses Archiv, Bd. XXXVI, Hft. 2) ein neues Verfahren empfohlen und auch mit Erfolg an der Lebenden exprobt. Er leitet die Fadenschlingen des Stumpfes durch den Wundtrichter nach unten in die Scheide, stülpt durch Anziehen der Fäden die Wundränder ein und vereinigt darïber das Bauchfell durch eine quere, beziehungsweise durch eine Tabaksbeutelnaht. Beaucamp. rühmt dem Verfahren Einfachheit, sicheren Abschluss der Bauchhöhle und kurze Heilungsdauer nach; er glaubt, dass man die nach dieser Methode operirten Frauen bei glattem Verlaufe nach 14 Tagen als geheilt ansehen dürfe. Das wäre allerdings ein grosser Vortheil dem alten Verfahren gegenüber. Ganz so einfach, wie Bea u camp meint, scheint mir aber die Frank'sche Methode doch nicht $z u$ sein, allein schon ein weites Heraufreichen der Blase dieselbe kann ja so weit nach oben gezogen sein, dass es überhaupt nur mit Mühe gelingt, einen Schlauch unter ihr anzulegen - muss die Umstülpung der Wundränder verhindern. Dazu kommt noch, dass das Verfahren, wenigstens bei Carcinomen, nicht ganz ungefährlich ist. Mag man die Stümpfe von oben nach unten durchleiten oder sie von der Scheide aus anziehen, immer muss das benutzte Instrument durch die Krebsmassen hindurch, und dabei ist eine Verschmierung derselben in den Stumpf sehr wohl denkbar. Trotzdem verdient nach den beiden günstigen Beobachtungen, welche Beaucamp beschrieb, das Verfahren in geeigneten Fällen Beachtung. und Nachpriifung.

In der letzten Zeit hatte ich zwei Mal Gelegenheit, an der Leiche die Veränderungen der Geschlechtstheile zu. sehen, welche sich nach Vornahme des erhaltenden Kaiserschnittes einstellen. Es handelte sich um die Frauen R. (Nr. XXVII) und M. (Nr. XXVI). Wie erwähnt, war Frau R. am siebenten Tage nach der Vornahme des erhaltenden. Kaiserschnittes infolge einer Blutung in die Bauchhöhle gestorben. Die $12 \mathrm{~cm}$ lange Gebärmutter, deren Wunde durch eine überall fest schliessende Naht vereinigt war, war nirgends verwachsen. Ueber die Narbe vergl. Leopold a. gen. Orte. Die Knoten der Fäden ragten frei in die Bauchböhle hinein, von irgend welcher Aufsaugung war nichts zu sehen. Kurze Zeit nach dieser einen Beobachtung kam die Frau M. zur Section. Dieselbe hatte im Februar dieses Jahres im vollsten Wohlsein die Klinik verlassen. Nach einiger Zeit bemerite sie, dass die auf dem Kreuzbeine be- 
findliche Geschwulst zu wachsen begann und ihr Schmerzen verursachte; letztere nahmen immer mehr zu und veranlassten die Frau Mitte August, ein Krankenhaus aufzusuchen. Hier wurde die faustgrosse, höckerige, ,lipomartige“ Geschwulst mit dem Perioste des Kreuzbeines entfernt; das darunter liegende Kreuzbein erschien cariös, und wurde deshalb die Frau behufs weiterer Operation dem hiesigen städtischen Krankenhause zugeführt. Als hier zum Zwecke der weiteren Untersuchung mit dem scharfen Löffel Stückchen von der Geschwulst abgeschabt wurden, entleerte sich plötzlich Liquor cerebro-spinalis aus dem eröffneten Rückenmarkscanale; von einer weiteren Operation wurde Abstand genommen. Am 8. October erfolgte unter meningitischen Erscheinungen der Tod. Herr Obermedicinalrath Dr. Stelzner hatte die Güte, uns zu der von Herrn Prof. Dr. Neelsen vorgenommenen Leichenöffnung einzuladen und uns die Organe der Frau zur weiteren Untersuchung zu überlassen. Aus dem Leichenberichte hebe ich kurz hervor: Tuberkulöse Caries des Kreuzbeines, alter Psoasabscess rechts. Tuberkulose der im kleinen Becken befindlichen Lymphdrüsen, tuberkulöse Meningitis basilaris et spinalis. Lungen frei, nur in dex rechten Spitze eine kleine Schwiele. Braune Herzatrophie, Verfettung der Leber, geringe Milzschwellung, chronisebe Blasenentzündung, Abscesse in den Nieren, rechtsseitige Hydronephrose.

Auf die Veränderungen des Beckens gehe ich an dieser Stelle weiter nicht ein, nur die der inneren Geschlechtstheile mögen genauer beschrieben werden (vergl. Fig. 1). Die Gebärmutter liegt mit ihren Anhängen an richtiger Stelle im kleinen Becken. Vom unteren freien Rande des grossen Netzes zieht ein feiner Verwachsungsstrang von etwa $15 \mathrm{~cm}$ Länge bis zum Grunde der Gebärmutter, wo er mit dem oberen Winkel der Kaiserschnittsnarbe verwachsen ist. Die Gebärmutter ist klein, atrophisch, hat eine Länge von 6 , eine Breite von $4 \frac{1}{2}$ und eine Dicke von $2 \mathrm{~cm}$. Der Douglas'sche Raum ist frei von entzündlichen Verbindungssträngen und bildet eine sehr tiefe Tasche zwischen den stark vorspringenden Douglas'schen Falten. Die Gebärmutter ist mit der vorderen Bauchwand nicht verwachsen - in der Mehrzahl der früheren Kaiserschnitte konnte man bei der Untersuchung der Lebenden eine derartige feste Verwachsung nachweisen -, zahlreiche Verbindungsbrücken erstrecken sich von der Gebärmutter zur hinteren Blasenwand; eine besonders breite nimmt an der Gebär- 
328 Münchmeyer, Beitrag zum Kaiserschnitte u. über das Verhalten

mutternarbe ihren Ursprung und breitet sich fächerförmig nach der Blase hin aus; eine zweite, weniger breite, findet sich an der Fig. 1. B.V.

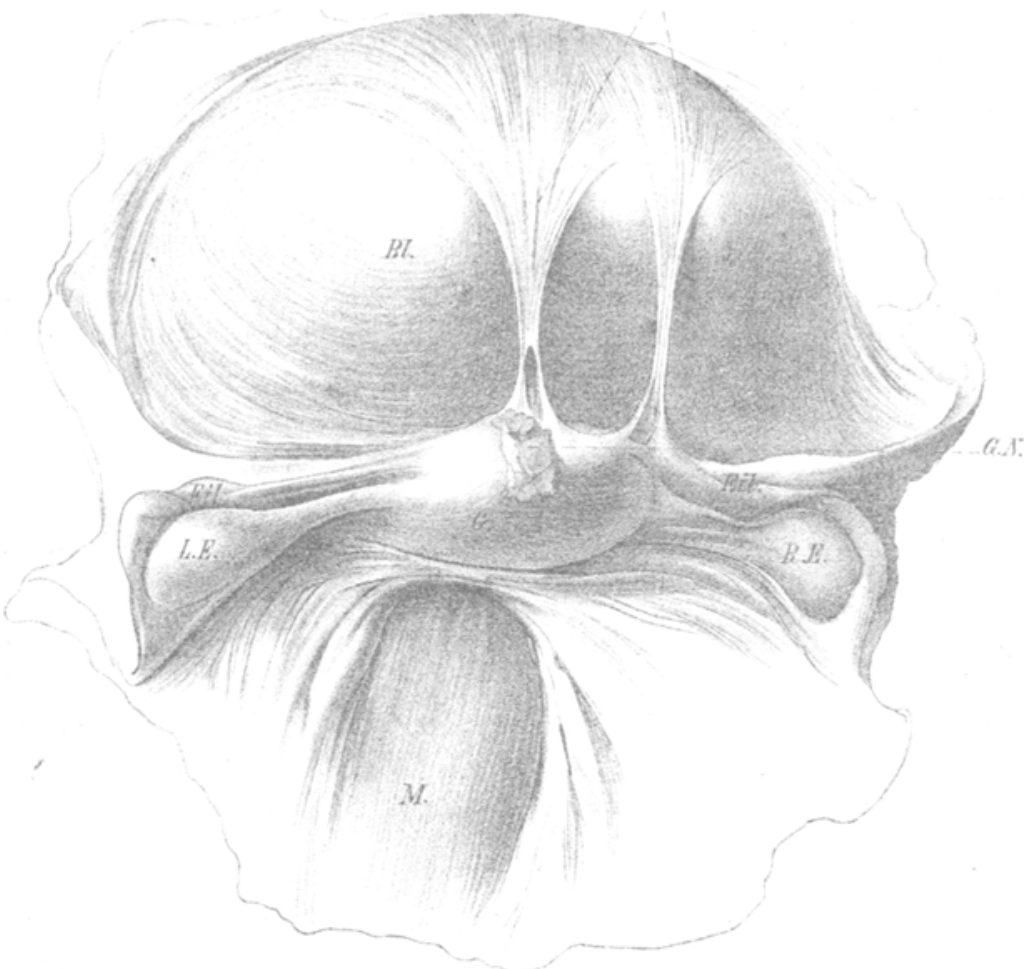

$B l .=$ Harnblase. $M I .=$ Mastdarm. $G .=$ Gebärmutter. $L . E$. und $R . E .=$ linker bez, rechter Eierstock. Eil. $=$ Eileiter. $G . N .=$ Gebärmutternarbe. $B . V .=$ Bauchfellverwachsungen.

Fig. 2.

C.F.

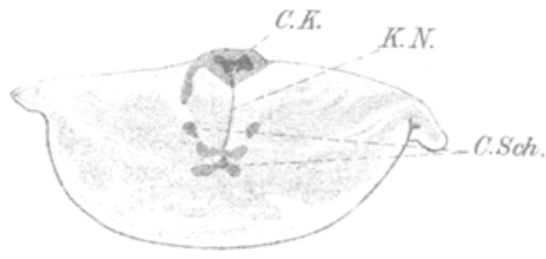

Querschnitt im oberen Drittel.
Fig. 3.

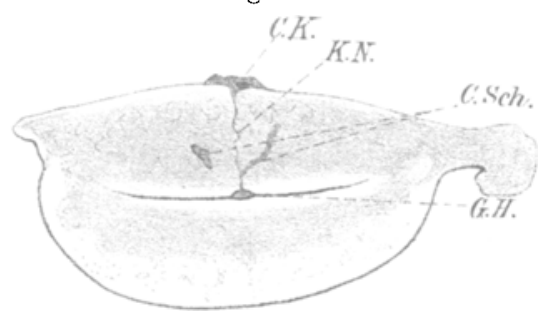

Querschnitt in der Mitte der Gebărmutter.

C. $K .=$ Catgutknoten. C. Sch. $=$ Catgutschlingen. $\quad G . H .=$ Gebärmutterböhle. $K . N .=$ Kaiserschnittsnarbe. 
Einmündung des linken Eileiters; zahlreiche feine, bei der Herausnahme der Gebärmutter zumeist zerrissene Fädchen verbinden Gebärmutter und Blase sowohl, als auch den linken Eierstock und linken Eileiter (auf der Abbildung sind nur die stärkeren Stränge gezeichnet). Dort, wo sich die breite Verbindungsbrücke befindet, beginnt am Gebärmuttergrunde die feine Narbe, nicht ganz $5 \mathrm{~cm}$ lang. Unter dem glatt darüber hinziehenden Bauchfelle - nur wo die Verwachsung mit dem Netze beginnt, ist dasselbe unregelmässig verdickt - sieht man die noch vollkommen erhaltenen Knoten der Chromsäurecatgutfäden durchschimmern. Dieselben sind, entsprechend der Verkürzung der Narbe, dicht aneinander herangeschoben worden. Die Eierstöcke sind klein, im linken findet sich ein kirschkerngrosser, mit geronnenem Blute gefüllter, nicht geplatzter Follikel. Auf Querschnitten durch die Gebärmutter sieht man die feine, linienförmige Narbe von aussen bis zum Spalte der Gebärmutterhöhle hinziehen; auch hier erscheinen deutlich die erhaltenen Schlingen der Chromcatgutfäden, während man mit dem blossen Auge von den Seidenfäden nichts entdecken kann (s. Fig. 2 u. 3).

Durch die ganze Dicke der Gebärmutter gelegte mikroskopische Schnitte zeigen folgendes Bild: Die Serosa zieht ganz gleichmässig über die Narbe fort, wird durch die Knoten der Fäden vorgebuchtet und erscheint hier bedeutend veidickt, schwielig. Die Narbe ist gekennzeichnet durch eine Schicht zellreichen, welligen Bindegewebes mit sehr langen Fasern; diese Bündel ziehen ununterbrochen von der Serosa bis zur Schleimhaut hin. Strotzend gefüllte Blutgefässe geben dem Schnitte einen fast cavernösen Bau; dieser Blutreichthum ist um so auffallender, als das Gewebe der Gebärmutter einen ausgesprochen inbrösen Charakter angenommen hat.

Von besonderem Interesse war es, nachzuweisen, ob und welche Veränderungen das Catgut und die Seide, sowie das sie umgebende Gewebe selbst so lange Zeit nach der Operation erlitten hatten. ES gelang; nach Einbettung $1 / 2 \mathrm{~cm}$ dicker Scheiben der ganzen Gebärmutterdicke in Celloidin genügend feine Mikrotomschnitte herzustellen, welche sowohl die durchschnittenen Knoten der Chromsäurecatgutfäden, als auch die Seidenfäden enthielten -, mit blossem Auge war von letzteren nichts mehr wahrnehmbar.

Thomson berichtete im 24. Hefte des Centralblattes für Gynäkologie (Jahrgang 1889) ïber Versuche, welche er an Thieren 
mit den verschiedenen Nahtmaterialien anstellte, die beim Kaiserschnitte besonders bevorzugt werden. Dabei fand er, dass Chromsäurecatgut, nach Mikulicz vorbereitet (auch wir verwandten bisher nur dieses), noch nach 64tägigem Liegen in der Gebärmutter vollständig erhalten war; gestützt werden diese Versuche durch die Beobachtung von Sänger und von Döderlein ${ }^{1}$, welche ganz erhaltene Chromcatgutfäden noch nach Monaten ausgestossen sahen. Thomson fand ferner, dass Seidenfäden nach 50tägigem Liegen in thierischen Geweben in ihren oberflächlichen Theilen aufgesogen, in den übrigen leicht zerreisslich waren. Wie decken sich diese Ergebnisse mit unseren Befunden?

Bei Fall R., sieben Tage nach der Operation zeigten sich die ganz unveränderten Chromcatgutfäden von einem ziemlich breiten Ringe von weissen Blutkörperchen umgeben, dadurch gleichsam von dem übrigen Gewebe abgekapselt. Die Fäden selbst waren, wie Schnitte frisch bereiteten Catguts bewiesen, ganz unverändert. Die allerdings viel dünneren Seidenfäden erschienen aufgelockert auch hier wurden zum Vergleiche unveränderte Fäden herangezogen -, nicht nur um, sondern auch zwischen sämmtlichen Fasern fanden sich sehr zahlreiche ausgewanderte Blutkörperchen. Dass dadurch eine leichtere Aufsaugung möglich wird, ist verständlich. Entsprechend der längeren Zeit nach der Operation waren die Befunde bei dem Falle M. (neun Monate) etwas anders, aber doch sehr ähnlich. Die Catgutfäden hatten nicht mehr die ursprüngliche Dicke, schienen auch an einzelnen Stellen schollig zu zerfallen, waren aber doch in ihrer Form - von einer Structur kann ja nicht die Rede sein - ziemlich erhalten. Nur in verhältnissmässig wenigen Schnitten liessen sich Reste von Seidenfäden nachweisen; dort, wo sie sich fanden, waren sie unverändert, runde, gleichmässige Fasern. Das Gewebe selbst hatte sich mit dem ihm einverleibten Fremdkörper ziemlich abgefunden; sowohl Catgut- als Seidenfäden waren vơn einem sehr kernhaltigen Bindegewebslager eingeschlossen; in letzterem und nach aussen von diesem fanden sich zahlreiche, den Farbstoff stark aufnehmende weisse Blutkörperchen.

Es decken sich somit die ron Thomson gemachten Befunde mit den unsrigen ziemlich genau, die Chromsäurecatgutfäden waren noch fast vollständig erhalten, die Seidenfäden, wenn auch nicht

1) Centralblatt für Gynäkologie 1889, Hft. 6 . 
ganz, so doch zum grösseren Theile aufgesogen; dabei darf man nicht ausser Acht lassen, dass letztere anfänglich nur ein Drittel der Dicke der Chromsäurefäden hatten.

Soll man daraus schliessen, dass das Chromsäurecatgut, mit dem in der Dresdner Klinik bisher so sehr gute klinische Erfolge erzielt wurden, zu verwerfen und durch die Seide zu ersetzen sei? Ich glaube, das wäre falsch.

Für den Kaiserschnitt wird dasjenige Nahtmaterial stets das beste sein, welches die Möglichkeit einer weiteren Schwangerschaft nicht hindert, bei vollkommener Asepsis so lange unverändert bleibt, als bis die Narbe ihre genügende Festigkeit erhielt, und dann möglichst bald aufgesogen wird. Diesen Forderungen entspricht aber weder das Sublimat-, noch das Chromsäurecatgut, ebenso wenig aber auch die Seide, um von anderen starren Nahtmaterialien gar nicht zu reden. Das Sublimatcatgut wird zu früh, das Chromsäurecatgut bisheriger Zubereitung, sowie die Seide sehr spät aufgesogen. Das nach Mikulicz bereitete Chromsäurecatgut liegt fünf Stunden in Chromsäure; dadurch erhält es seine grosse Härte und Widerstandsfähigkeit, es wird gleichsam gegerbt; das ist ein Nachtheil, der ihm anhaftet. Es muss versucht werden, ein Chromsäurecatgut mit gleich vorzüglicher aseptischer Beschaffenheit, doch nach kürzerem Liegen in Chromsäure - zwei Stunden oder noch weniger - herzustellen, welches genug, um nicht zu früh, und nicht zu stark gegerbt ist, um nicht zu spät aufgesogen zu werden. Das müssen weitere Versuche und Erfahrungen lehren. ${ }^{1}$ )

Meinem hochgeehrten Chef, Herrn Obermedicinalrath Prof. Dr. Leopold, sage ich auch an dieser Stelle meinen wärmsten Dank für die Anregung zu dieser Arbeit, die er mit freundlichem Rathe förderte.

1) Leider konnten die kürzlich erschienenen Arbeiten von Brunner (Beiträge zur klinischen Chirurgie, Bd. VI) und Döderlein (Münchener medicinische Wochenschrift, Nr. 4) nicht mehr berücksichtigt werden, da die vorliegende Arbeit schon vor einiger Zeit zum Drucke übergeben wurde. 